\title{
High GP73 expression correlates with poor response to neoadjuvant chemotherapy and survival in gastric cancer
}

\section{Jian Guo Shen}

Zhejiang University School of Medicine Sir Run Run Shaw Hospital

Jun Shen

Zhejiang University School of Medicine Sir Run Run Shaw Hospital

Rong Yue Teng

Zhejiang University School of Medicine Sir Run Run Shaw Hospital

\section{Lin Bo Wang}

Zhejiang University School of Medicine Sir Run Run Shaw Hospital

\section{Wen He zhao}

Zhejiang University School of Medicine Sir Run Run Shaw Hospital

Qin Chuan Wang ( $\triangle$ wqctiger@zju.edu.cn )

\section{Research}

Keywords: GP73, gastric cancer, prognosis, neoadjuvant chemotherapy

Posted Date: March 24th, 2020

DOI: https://doi.org/10.21203/rs.3.rs-18930/v1

License: (9) This work is licensed under a Creative Commons Attribution 4.0 International License. Read Full License 


\section{Abstract}

Background囚Golgi protein 73 (GP73) is a type II Golgi transmembrane protein which is over expressed in several cancers, however, its role in gastric cancer is still unclear. The aim of this study is to investigate if high GP73 expression is associated with pathological tumor response to neoadjuvant chemotherapy and prognosis for patients with gastric cancer.

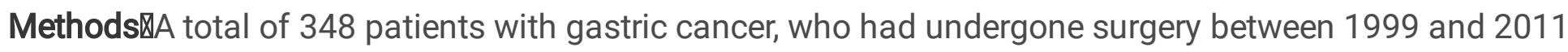
were retrospectively reviewed, GP73 expression was examined in tumor tissues using tissue microarray (TMA), the correlations between its expression and pathological response to neoadjuvant chemotherapy as well as patient prognosis were analyzed.

Results $\llbracket$ We found that GP73 expression was not associated with clinicopathologic features including tumor size, differentiation and TNM stage. High expression of GP73 was associated with less pathological tumor response to neoadjuvant chemotherapy and poor survival in gastric cancer, multivariate analysis showed GP73 expression was an independent predictor for pathological response to neoadjuvant chemotherapy and for prognosis in patient with gastric cancer.

Conclusions囚Our results suggest that GP73 is a promising biomarker to identify patients with poor prognosis as well as a promising predictor for the effect of neoadjuvant chemotherapy in gastric cancer.

\section{Background}

Gastric cancer (GC) is now the fourth most common cancer and is the second most common cause of death from cancer in the world. It is estimated that two-thirds of gastric cancer cases occur in developing countries and $42 \%$ in China alone [1, 2]. Surgery remains the first choice of treatment for GC. However, the 5 -year survival rate for patients with all stages stays 20\% [3]. Thus, it is essential to develop effective prognostic factor that may predict patient survival in gastric cancer.

Preoperative chemotherapy is now widely used in patient with locally advanced gastric cancer, since it can improve complete surgical resection so as to improve survival [4]. However, the effect of neoadjuvant chemotherapy stays at $50 \%$, in unresponsive cases, it may have potential to delay surgical treatment [5]. therefore, tailored therapy may be conducted if factors predicting the response of neoadjuvant chemotherapy can be preoperatively identified in gastric cancer.

GP73, also known as GOLM1 and GOLPH2, is a type II Golgi protein which is normally located within the cis-Golgi complex [6]. Most studies showed that GP73 was over-expressed and was correlated with tumor progression and poor survival in patients with several types of cancer including hepatocellular carcinoma, colon cancer [7, 8]. The association between GP73 and gastric cancer remains controversial, GP73 was reported to be correlated with tumor differentiation in gastric cancer [9], however, other study found the correlation between GP73 and patient survival [10]. 
In this study, we evaluated GP73 expression by immunohistochemistry (IHC) on a tissue microarray containing 348 gastric cancer tumor tissues, and try to investigate the expression of GP73 and its relationship with pathological response in patients with neoadjuvant chemotherapy as well as prognosis in patient with gastric cancer.

\section{Materials And Methods}

\section{Patients and tissue samples}

A total of 348 patients with gastric cancer who underwent surgical treatment in the department of surgical oncology, Sir Run Run Shaw hospital between 1996 and 2011 were enrolled into the study. Patient demographics and clinicopathologic characteristics, including tumor size, location, depth of invasion, tumor differentiation, node status and TNM stage were documented. Patient with clinically staged as T2 and above or lymph node metastasis were recommend to have neoadjuvant chemotherapy, the effect of chemotherapy was assessed based on the RECIST criteria. Surgical treatment was generally performed according to the rules of the Japanese Research Society for Gastric Cancer. After surgery, tumor specimens were send to the Pathology and the pathologic stage was determined according to the rules of the 6th edition of UICC and the stage grouping of the UICC/AJCC. Pathological response was evaluated among the patients with neoadjuvant chemotherapy by using tumor regression grade (TRG), which was proposed by Mandard's et al [11]. Briefly, cancer with complete regression was graded as TRG4, isolated cell nests as TRG 3, more residual cancer cells but fibrosis still predominates as TRG 2 and residual cancer outgrowing fibrosis or absence of regressive changes as TRG 1. Generally, TRG 3 and 4 was classified as tumor regression, which was referenced in previous studies. The patients were followed up until death or until the date of last follow-up of Feb 28, 2015, and 179 patients (51.4\%) died during the follow up period.

\section{TMA preparation and IHC}

GP73 expression was evaluated in 348 gastric cancer tissues by IHC in TMA. The construction of TMA was previously reported [12]. The 5- $\mu \mathrm{m}$-thick slices of TMA were prepared and stored at $4{ }^{\circ} \mathrm{C}$ until use. GP73 was IHC stained in the TMA, The staining condition was validated using negative and positive tissue controls for GP73 antibodies.

\section{Interpretation of IHC GP73 expression on TMA}

The IHC staining of cytoplasmic GP73 were evaluated by two pathologists independently, based on the intensity and the proportion of positively stained cancer cells. The proportion of positively stained cancer cells was scored as follows: 0 for no positive tumor cells, 1 for $<10 \%$ positive tumor cells, 2 for $10-35 \%$ positive tumor cells, 3 for $35-70 \%$ positive tumor cells and 4 for $>70 \%$ positive tumor cells. The intensity was scored as: 0 (no staining), 1 (weak staining), 2 (moderate staining) and 3 (strong staining). Scores for intensity and proportion of positive cells were multiplied as final histoscores of the samples. Scores $\leq$ 3 was regarded as tumors with low or none GP73 expression and scores $\geq 4$ as high GP73 expression. 


\section{Statistical analysis}

All demographic data, clinicopathological variables and IHC results were coded and entered into a gastric cancer database. Double data entry and logic checks were used for error reduction. All statistical analyses in the study were performed by SPSS 22.0 (SPSS, Chicago, IL, USA). The Chi-square test and Fisher's exact test were performed to assess the correlations between GP73 expression level and clinical characteristics. Kaplan-Meier method was used to calculate the survival probabilities and log rank test was used to compare survival curves. Independent factors influencing the survival were determined by multivariate analysis using cox regression model. Factors predicting neoadjuvant pathological response were determined by means of logistic regression analysis. A p value of less than 0.05 was considered statistically significant.

\section{Results}

\section{Correlation between GP73 expression and clinicopathologic parameters}

GP73 expression was determined by IHC in 348 gastric cancer tissues on the TMA. GP73 was mainly localized in the cytoplasm of the cancer cells (Fig. 1). A total of 152/348 gastric cancer tissues (43.7\%) exhibited high GP73 expression, while 196/348 (56.3\%) observed low or none GP73 expression. the correlations between GP73 expression and clinical characteristics are shown in Table 1. Patient with larger tumor size was more likely to have high GP73 expression than those with small tumor size $(\mathrm{P}=$ 0.014), however, other clinicopathologic parameters including age, tumor location, differentiation, peritoneal metastasis and TNM stage were not associated with the GP73 expression level. 
Table 1

Correlation between GP73 expression and clinicopathologic factors of 348 gastric cancer patients

\begin{tabular}{|c|c|c|c|}
\hline \multirow[t]{2}{*}{ Characteristics } & \multicolumn{2}{|l|}{ GP73 } & \multirow[t]{2}{*}{ P-value } \\
\hline & $\begin{array}{l}\text { Low or none } \\
\text { No. of cases (\%) }\end{array}$ & $\begin{array}{l}\text { High } \\
\text { No. of cases }(\%)\end{array}$ & \\
\hline Age (Mean $\pm S D$, years) & $58.1 \pm 11.2$ & $60.3 \pm 12.9$ & 0.087 \\
\hline Gender & & & 0.396 \\
\hline Female & $58(54.2)$ & $49(45.8)$ & \\
\hline Male & $132(56.4)$ & $102(43.6)$ & \\
\hline Tumor location & & & 0.303 \\
\hline Proximal & $33(53.2)$ & $29(46.8)$ & \\
\hline Middle & $48(64.0)$ & $27(36.0)$ & \\
\hline Low body & $98(52.1)$ & $90(47.9)$ & \\
\hline Whole & $6(66.7)$ & $3(33.3)$ & \\
\hline Tumor size (Mean \pm SD, $\mathrm{cm}$ ) & $5.1 \pm 2.7$ & $5.8 \pm 2.5$ & 0.014 \\
\hline Differentiation & & & 0.898 \\
\hline Well or moderate & $56(57.1)$ & $42(42.9)$ & \\
\hline Poor or no & $94(55.6)$ & $75(44.4)$ & \\
\hline T classification & & & 0.094 \\
\hline $\mathrm{T} 1+\mathrm{T} 2$ & $57(62.6)$ & $34(37.4)$ & \\
\hline $\mathrm{T} 3+\mathrm{T} 4$ & $132(53.9)$ & $113(46.1)$ & \\
\hline LN metastasis & & & 0.236 \\
\hline No & $54(60)$ & $36(40)$ & \\
\hline Yes & $131(54.8)$ & $108(45.2)$ & \\
\hline Peritoneal Metastasis & & & 0.392 \\
\hline No & $191(56)$ & $150(44)$ & \\
\hline Yes & $2(40)$ & $3(60)$ & \\
\hline TNM stage & & & 0.298 \\
\hline
\end{tabular}




\begin{tabular}{|c|c|c|c|}
\hline \multirow[t]{3}{*}{ Characteristics } & \multicolumn{2}{|l|}{ GP73 } & \multirow[t]{3}{*}{ P-value } \\
\hline & Low or none & High & \\
\hline & No. of cases $(\%)$ & No. of cases $(\%)$ & \\
\hline$|+| \mid$ & $83(58.5)$ & $59(41.5)$ & \\
\hline III + IV & $106(54.9)$ & $87(45.1)$ & \\
\hline Neural invasion & & & 0.500 \\
\hline No & $170(55.7)$ & $135(44.3)$ & \\
\hline Yes & $24(57.1)$ & $18(42.9)$ & \\
\hline Carcinomatous nodule & & & 0.148 \\
\hline No & $171(57.2)$ & $128(42.8)$ & \\
\hline Yes & $23(47.9)$ & $25(52.1)$ & \\
\hline Surgical type & & & 0.075 \\
\hline Radical & $157(58.8)$ & $110(41.2)$ & \\
\hline Palliative & 37 (48.7) & 39 (51.3) & \\
\hline
\end{tabular}

\section{Correlation of GP73 expression with patient overall survival}

Univariate analysis was conducted to investigate the relationship between the clinicopathologic characteristics and patients overall survival, patient with high GP73 expression observed a shorter median overall survival than those with low or none GP73 expression (Fig. 2). Significant correlations were also observed between groups in terms of patient age, tumor location, size, differentiation, neural invasion, TNM stage and surgical type.

Multivariate analysis using the Cox proportional hazard model was employed to identify the prognostic factors in gastric cancer patient. High GP73 expression was found to be one of the independent prognostic factors for survival in gastric cancer patients (Table 2). 
Table 2

Multivariate analysis of various prognostic parameters in patients with gastric cancer using cox regression model

\begin{tabular}{|llll|}
\hline Clinicopathologic factors & HR & $95 \%$ Cl & P value \\
\hline Age (<60 vs. >=60 years) & 1.024 & $1.005-1.043$ & 0.015 \\
\hline Tumor location (P/W vs. M/L) & 0.862 & $0.656-1.133$ & 0.288 \\
\hline Differentiation ( well vs. poor) & 1.458 & $0.900-2.360$ & 0.125 \\
\hline Tumor size (<4 vs.>4 cm) & 1.051 & $0.980-1.128$ & 0.164 \\
\hline TNM stage ( I,Il vs. III,IV) & 2.657 & $1.669-4.230$ & 0.000 \\
\hline Neural invasion (no vs. yes) & 2.560 & $1.523-4.304$ & 0.000 \\
\hline GP73 expression (low and none vs. high) & 1.593 & $1.035-2.452$ & 0.034 \\
\hline P/W: proximal/whole body; M/L: middle/low body & & \\
\hline
\end{tabular}

\section{Efficacy of neoadjuvant chemotherapy in low or none- and high GP73 expressing patients}

Forty-three out of 348 patients (12.4\%) with locally advanced gastric cancer were conducted neoadjuvant chemotherapy before surgery, among them, $74.4 \%$ patient treated with oxaplatin-based regimens and the others $(25.6 \%)$ with docetaxol-based regimens, and $55.8 \%$ patients completed more than three cycles of neoadjuvant chemotherapy before surgery. Finally, $74.4 \%$ patients performed surgery radically.

Pathologic tumor response after neoadjuvant chemotherapy was routinely assessed by the pathologist by using TRG. TRG1 was observed in 17 patients (39.5\%), TRG2 in 16 patients (37.2\%), TRG3 in 8 patients (18.6\%) andTRG4 in 2 patients (4.7\%), respectively. Ten patients (23.3\%) showed tumor regression (TRG 3 or 4 ) as defined in the present study, and 2 patients showed complete regression.

The relationship between neoadjuvant pathological response and clinical variables was evaluated using univariate analysis, tumor size and GP73 expression was shown to be significantly associated with tumor regression grade. Patient with high GP73 expression represented less tumor regression as compared to those with low or none GP73 expression ( $p=0.023$ ) (Fig. 3). Multivariate analysis using Logistic regression model identified GP73 as one of the independent predictive factor for pathological response $(p=0.045$, Odds Ratio, $10.647,95 \% \mathrm{Cl}$ for odds ratio, $1.050-107.954)$ (Table 3 ). 
Table 3

Multivariate logistic analysis to identify predictors of tumor regression in patients with neoadjuvant chemotherapy

\begin{tabular}{|llll|}
\hline Variables & Odds ratio & $\mathbf{9 5 \%} \mathbf{C l}$ & P value \\
\hline GP73 expression (High vs. low or none) & 10.647 & $1.050-107.954$ & 0.045 \\
\hline Tumor size $(\mathrm{cm})$ (continuous) & 1.494 & $1.060-2.108$ & 0.022 \\
\hline
\end{tabular}

\section{Discussion}

In the present study, we found that high GP73 expression was associated with poor outcome in patients with gastric cancer and was an independent prognostic factor for survival. To the best of our knowledge, this is the first study to evaluate the correlation between GP73 expression and pathological response to chemotherapy in gastric cancer. Patients with high GP73 expression were more likely to obtain less pathological tumor regression as compared to patients with low or none GP73 expression, these results indicate GP73 as a potential clinical marker to predict pathological tumor response in advanced gastric cancer receiving neoadjuvant chemotherapy.

GP73 was originally cloned from a library derived from the liver tissue of a patient with adult giant-cell hepatitis [13]. The role of GP73 are mainly studied in hepatocellular carcinoma, high-expression of GP73 was associated with tumor size, differentiation, grade and survival, which indicate that GP73 is a valuable marker using as an independent diagnostic tool for hepatocellular carcinoma [14-18]. However, knowledge on the function of GP73 is limited in gastric cancer.

The relationship between GP73 expression and gastric cancer progression is still controversial. A study from Chen et al. assessed GP73 protein expression by immunohistochemistry in both tumor and nontumorous gastric mucosal tissue, they found GP73 was down-regulated in gastric cancer, and its expression in gastric cancer was associated with tumor differentiation [9]. Liu et al's study revealed a significant correlation between GP73 expression and clinical stage, lymph node metastasis and venous invasion, thus the study made a conclusion that GP73 expression may be associated with tumor progression [10], However, in the present study, no significant correlation was found between GP73 expression and clinical variables including age, differentiation and TNM stage, this discrepancy may be explained as the following: firstly, a comparatively more gastric cancer samples was enrolled in the present study, the difference of patient clinical characteristics as a selection bias may influence the analysis. Secondly, we assessed GP73 expression by IHC on TMA, and it may, to some extent, reduce the experimental bias as compared to IHC on a separate paraffin tissue. Finally, the difference of interpretation of GP73 IHC results may also effect the results we achieved.

The prognostic effect of GP73 expression in some cancers was reported. A study from Jiang et al showed that patients with high GP73 expression achieved poorer outcome than those with low or none GP73 expression in hepatocellular carcinoma (HCC) [17], however, Sun et al's reports showed no survival 
difference between both groups in terms of GP73 expression [18]. In the present study, we observed that patients with higher GP73 expression have a significantly lower overall survival rate than those with low or none GP73 expression, and multivariate analysis revealed GP73 expression was an independent predictor for survival. These results were in accordance with a study of Liu et al, who analyzed the GP73 expression by IHC in 385 gastric cancer patients, and found that GP73 is a useful prognostic variable of overall survival in gastric cancer patients [10]. Our results, with previous reports, may indicate that GP73 may have the potential to be a new target in the treatment of gastric cancer.

A recent randomized phase III trial demonstrated a survival benefit for gastric cancer patients with perioperative chemotherapy when compared with surgery alone [4], and tumor regression grade was mainly utilized as a reasonable method for predicting pathologic response of cytotoxic agents [19], thus, it is essential to identify clinical markers predicting the pathologic tumor response before treatment. In the present study, we found that patients with high GP73 expression were more likely to obtain less tumor response than those with low or none GP73 expression, and further multivariate analysis found that GP73 expression serve as one of the important predictors of pathologic tumor regression, these results revealed that GP73 might be helpful for selecting treatment options in patients with gastric cancer.

The mechanism of the correlation between GP73 expression and chemotherapy response in cancer is unclear. Ye et al reported that GP73 may significantly change cell proliferation and apoptosis so as to influence the oxaplatin resistance in hepatic carcinoma cells [20]. Zhou et al's study found that GP73 induced cisplatin resistance in HT29 colon cancer cells was related to the activation of the mitogenactivated protein kinase/ERK and Wnt/ $\beta$-catenin signaling pathways [21]. However, the interaction between GP73 and chemoresistance in gastric cancer was rarely reported, and thus need to be further studied.

In conclusion, our results suggest that GP73 may serve as a promising biomarker to identify patients with poor prognosis and to predict the effect of neoadjuvant chemotherapy in gastric cancer, however, its potential role in the management of patient with gastric cancer should be validated in large-scale studies.

\section{Abbreviations}

GP73, Golgi protein 73; TMA, tissue microarray; GC, Gastric cancer; IHC, immunohistochemistry; TRG, tumor regression grade $\triangle \mathrm{HCC} \triangle$ hepatocellular carcinoma

\section{Declarations}

\section{Ethics approval and consent to participate}

This research was approved by the Institution Ethics Committee of Sir Run Run Shaw hospital.

\section{Consent for publication}


All participants signed informed consent.

\section{Availability of data and materials}

All data and materials are available.

\section{Competing interests}

The authors declare that they have no competing interests.

\section{Authors' contributions}

JGS and QCW were responsible for the conception, design, and acquisition of data, JS, RYT and LBW analyzed and interpreted the data. JGS wrote the final draft. All authors read and approved the final manuscript.

\section{Acknowledgements}

This study was supported by the grant of the Zhejiang National Science Funding (no. LY18H160005) and the Zhejiang Medical Association Clinical Research Fund Project (no. 2019ZYC-A02).

\section{Funding}

Not applicable

\section{References}

1. Jemal A, Siegel R, Xu J, Ward E. Cancer statistics, 2010. CA Cancer J Clin. 2010;60:277-300.

2. Chen W, Zheng R, Baade PD. Cancer statistics in China, 2015. CA Cancer J Clin. 2016;66(2):115-132.

3. Paoletti X, Oba K, Burzykowski T, et al. Benefit of adjuvant chemotherapy for resectable gastric cancer: a meta-analysis. JAMA. 2010;303: 1729-1737.

4. Cunningham D, Allum WH, Stenning SP, et al. Perioperative chemotherapy versus surgery alone for resectable gastroesophageal cancer. N Engl J Med. 2006;355:11-20.

5. Giralt J, Eraso A, Armengol M, et al. Epidermal growth factor receptor is a predictor of tumor response in locally advanced rectal cancer patients treated with preoperative radiotherapy. Int J Radiat Oncol Biol Phys. 2002;54:1460-1465.

6. Kladney RD, Bulla GA, Guo L, Mason AL, Tollefson AE, Simon DJ, Koutoubi Z, Fimmel CJ. GP73, a novel Golgi-localized protein upregulated by viral infection. Gene. 2000; 249:53-65.

7. Yang J, Li J, Dai W, et al. Golgi protein 73 as a biomarker for hepatocellular carcinoma: A diagnostic meta-analysis. Exp Ther Med. 2015;9(4):1413-1420.

8. Liang R, Liu Z, Piao X, et al. Research progress on GP73 in malignant tumors. Onco Targets Ther. 2018;11:7417-7421. 
9. Chen LG, Wang HJ, Yao HB, et al. GP73 is down-regulated in gastric cancer and associated with tumor differentiation. World Journal of Surgical Oncology. 2013;11:132.

10. Liu GL, Zhang Y, HE F, et al. Expression of GOLPH2 is associated with the progression of and poor prognosis in gastric cancer. Oncol Rep.2014; 32: 2077-2085.

11. Mandard AM, Dalibard F, Mandard JC, et al. Pathologic assessment of tumor regression after preoperative chemoradiotherapy of esophageal carcinoma. Clinicopathologic correlations. Cancer. 1994; 73: 2680-6.

12. Wang Q, Liu X, Zhou J, et al. Ribonucleotide reductase large subunit M1 predicts poor survival due to modulation of proliferative and invasive ability of gastric cancer. PLoS One. 2013;8(7):e70191.

13. Zhou Y, Li L, Hu L, Peng T. Golgi phosphoprotein 2 (GOLPH2/GP73/ GOLM1) interacts with secretory clusterin. Mol Biol Rep. 2011; 38:1457-1462.

14. Ai N, Liu W, Li ZG, Ji H, Li B, Yang G. High expression of GP73 in primary hepatocellular carcinoma and its function in the assessment of transcatheter arterial chemoembolization. Oncol Lett. 2017;14(4):3953-3958.

15. Bao YX, Yang Y, Zhao HR, et al. [Clinical significance and diagnostic value of Golgi-protein 73 in patients with early-stage primary hepatocellular carcinoma]. Zhonghua Zhong Liu Za Zhi. 2013;35(7):505-508.

16. Zhang S, Ge W, Zou G, et al. MiR-382 targets GOLM1 to inhibit metastasis of hepatocellular carcinoma and its down-regulation predicts a poor survival. Am J Cancer Res. 2018;8(1):120-131.

17. Jiang K, Li W, Shang S, et al. Aberrant Expression of Golgi Protein 73 Is Indicative of a Poor Outcome in Hepatocellular Carcinoma. Oncol Rep. 2016; 35 (4), 2141-50.

18. Sun YL, Yang HY, Mao Yl, et al. Increased Golgi Protein 73 Expression in Hepatocellular Carcinoma Tissue Correlates With Tumor Aggression but Not Survival. J Gastroenterol Hepatol. 2011; 26 (7), 1207-12.

19. Wang LB, Teng RY, Jiang ZN, et al. Clinicopathologic variables predicting tumor response to neoadjuvant chemotherapy in patients with locally advanced gastric cancer. J Surg Oncol. 2012;105(3):293-296.

20. Ye JZ, Yan SM, Yuan CL, et al. GP73 level determines chemotherapeutic resistance in human hepatocellular carcinoma cells. J Cancer. 2018;9(2):415-423.

21. Zhou ZP, Wang LP, Hong ZS, et al. Silencing GOLPH3 gene expression reverses resistance to cisplatin in HT29 colon cancer cells via multiple signaling pathways. Int J Oncol. 2018;53(3):1183-1192.

\section{Figures}



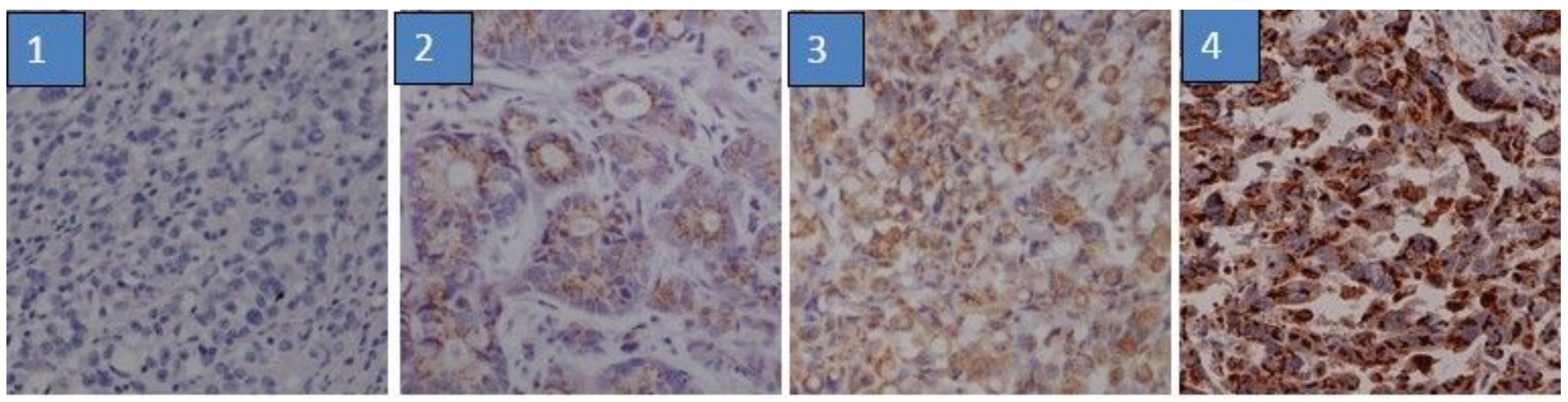

\section{Figure 1}

Immunohistochemistry staining of GP73 according to its expression. The IHC staining of GP73 in TMA was evaluated based on staining intensity and proportion of positively stained tumor cells. 1. negative staining (score 0 ); 2 , weak staining (score 1); 3 , medium staining (score 2); 4, strong staining (score 3 ).

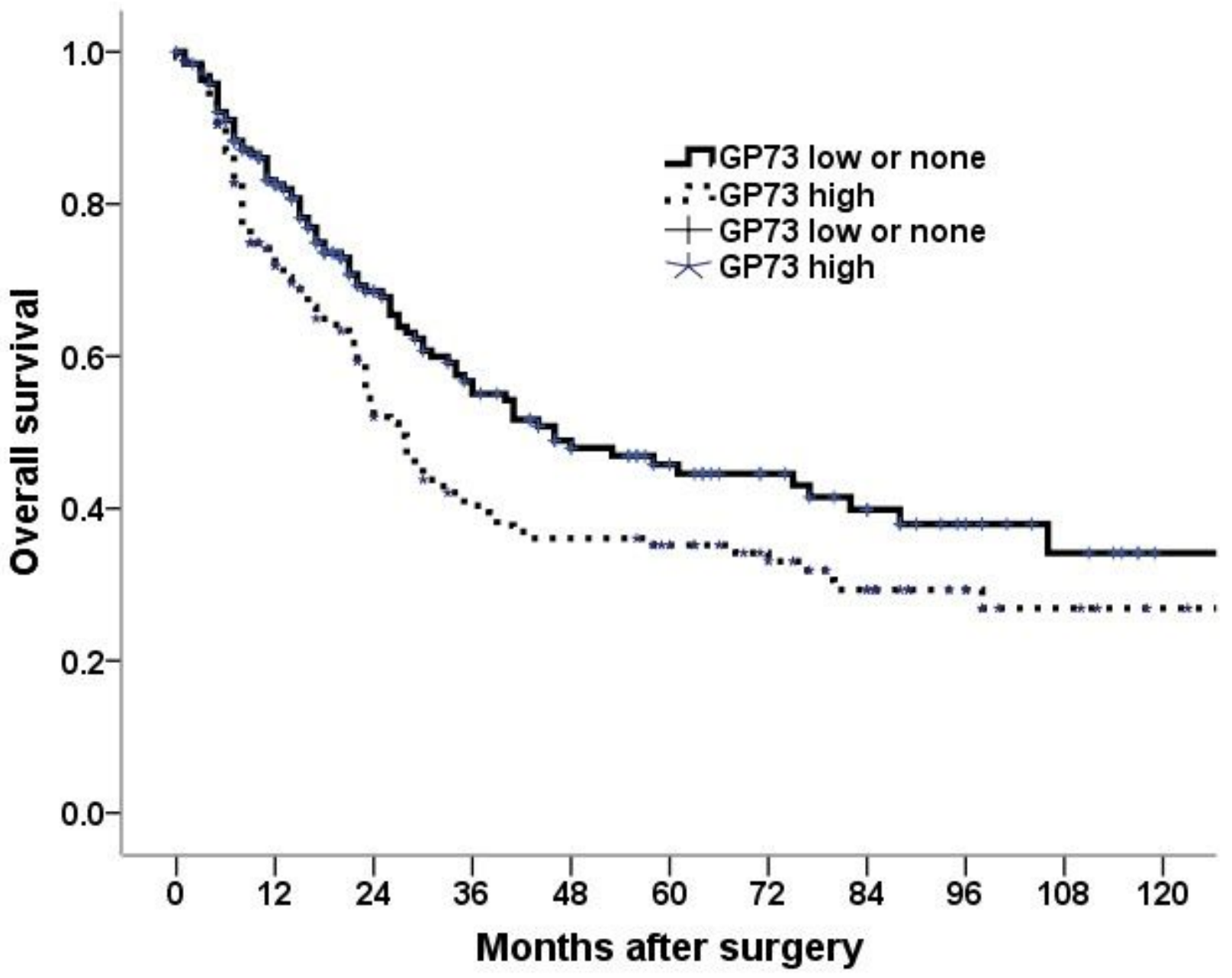

Figure 2

Kaplan-Meier survival analysis for 348 gastric cancer patients with a low and none versus a high GP73 expression tumors 


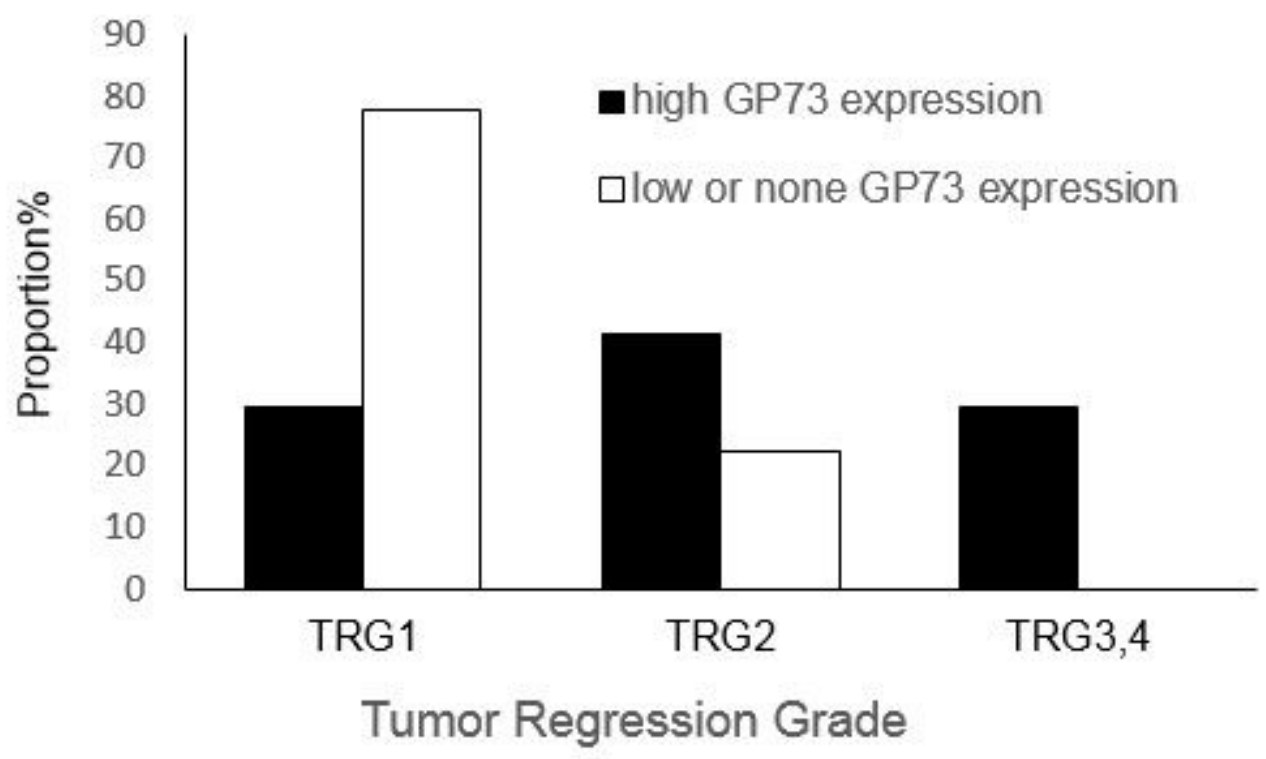

Figure 3

Correlation between GP73 expression and tumor regression grade in gastric cancer patient with neoadjuvant chemotherapy 